\title{
Temperature induced differences in the nanostructure of hot-wire deposited silicon-germanium alloys analyzed by anomalous small-angle $x$-ray scattering
}

\author{
G. Goerigk ${ }^{\mathrm{a})}$ \\ Institut für Festkörperforschung, Forschungszentrum Jülich, Postfach 1913, D-52425 Jülich, \\ Federal Republic of Germany \\ D. L. Williamson \\ Department of Physics, Colorado School of Mines, Golden, Colorado 80401
}

(Received 15 September 2005; accepted 16 December 2005; published online 27 April 2006)

\begin{abstract}
The nanostructure of hydrogenated amorphous silicon-germanium alloys, $a-\mathrm{Si}_{1-x} \mathrm{Ge}_{x}: \mathrm{H}$ $(x=0.62-0.70)$, prepared by the hot-wire deposition technique applying different substrate and filament temperatures was analyzed by anomalous small-angle x-ray scattering experiments. The pure-resonant scattering contribution, which is related to the structural distribution of the Ge component in the alloy, was separated from the total small-angle scattering for one sample series. For all alloys the Ge component was found to be inhomogeneously distributed. The shape of the pure-resonant and the mixed-resonant scattering curves reveal significant differences indicating the presence of a third phase, probably hydrogen clusters and/or voids. The thin films showed improved microstructure when lowering the filament temperature to $1800{ }^{\circ} \mathrm{C}$. Additional improvement was achieved by optimizing the substrate temperature (between 260 and $305^{\circ} \mathrm{C}$ ) resulting in suggested mass fractal structures of Ge with the fractal dimension $p<1.6$ and a size of about $40 \mathrm{~nm}$. The nature of the microstructural changes induced by changes in filament temperature compared to those induced by the changes in substrate temperature were clearly different. The improved microstructure of the alloys could be correlated with improved optoelectronic properties of the material. (C) 2006 American Institute of Physics. [DOI: 10.1063/1.2187088]
\end{abstract}

\section{INTRODUCTION}

Hydrogenated amorphous silicon-germanium alloys are used in solar cell technology, where the germanium is added to produce a lower band gap material to absorb the longer wavelength photons of the solar spectrum and to achieve higher efficiences. Previous small-angle $\mathrm{x}$-ray scattering (SAXS) and anomalous small-angle $\mathrm{x}$-ray scattering (ASAXS) studies revealed that, in addition to voids, nonuniformly distributed $\mathrm{Ge}$ contributes to the material inhomogeneities, ${ }^{1-4}$ which are strongly related to the degradation of the optoelectronic properties. In recent years there has been a growing interest especially in the hot-wire chemical-vapor depostion (HWCVD) technique ${ }^{5}$ due to the evidence of improved stability and improved optoelectronic properties of the material, as well as the potentially beneficial manufacturing feature of higher deposition rates than the current industrial technique of plasma-enhanced chemicalvapor deposition (PECVD). ${ }^{6,7}$ A group at National Renewable Energy Laboratory, U.S.A. (NREL) found evidence of improved photoresponse from HWCVD a-SiGe:H alloys with narrow bandgaps deposited at different filament temperatures, filament diameters, and optimized substrate temperatures. ${ }^{8,9}$ Here we report on ASAXS results from a series of narrow band gap films $[1.21 \mathrm{eV}$

\footnotetext{
${ }^{\text {a) }}$ Author to whom correspondence should be addressed; present address: c/o DESY-HASYLAB, Notkestrasse 85, D-22603 Hamburg, Federal Republic of Germany; FAX: +49-40-89984475; electronic mail: guenter.goerigk@desy.de
}

$<$ Tauc gap $\left.\left(E_{\text {Tauc }}\right)<1.32 \mathrm{eV}\right]$ made by HWCVD at different filament and substrate temperatures. ASAXS enables the structural characterization of inhomogeneously distributed $\mathrm{Ge}$ in the material. The distribution of Ge is not accessible by conventional SAXS measurements, because the scattering contributions of the Ge-related structures cannot be distinguished from those of other inhomogeneities caused by voids and/or hydrogen clusters.

\section{ASAXS MEASUREMENTS}

In the case of a ternary silicon-germanium-hydrogen alloy the scattering amplitude is

$$
\begin{aligned}
A(\mathbf{q})= & \int_{V_{p}} \Delta \rho_{\mathrm{H}}(\mathbf{r}) \exp (-i \mathbf{q r}) d^{3} r+\int_{V_{p}} \Delta \rho_{\mathrm{Ge}}(\mathbf{r}) \\
& \times \exp (-i \mathbf{q r}) d^{3} r,
\end{aligned}
$$

where $q$ is the magnitude of the scattering vector $[=(4 \pi / \lambda) \sin \Theta], 2 \Theta$ is the scattering angle, $\lambda$ the $\mathrm{x}$-ray wavelength, and $V_{p}$ is the irradiated sample volume. $\Delta \rho_{\mathrm{H}}, \Delta \rho_{\mathrm{Ge}}$ are the difference electron densities of the hydrogen and the Ge atoms,

$$
\Delta \rho_{\mathrm{H}}(\mathbf{r})=\Delta f_{\mathrm{H}} \cdot u(\mathbf{r})=\left(f_{\mathrm{H}}-\rho_{\mathrm{Si}} V_{\mathrm{H}}\right) u(\mathbf{r}),
$$




$$
\begin{aligned}
\Delta \rho_{\mathrm{Ge}}(\mathbf{r}, E) & =\Delta f_{\mathrm{Ge}}(E) v(\mathbf{r}) \\
& =\left[\left(f_{0, \mathrm{Ge}}-\rho_{\mathrm{Si}} V_{\mathrm{Ge}}\right)+f_{\mathrm{Ge}}^{\prime}(E)+i f_{\mathrm{Ge}}^{\prime \prime}(E)\right] v(\mathbf{r}),
\end{aligned}
$$

calculated from the electron density, $\rho_{\mathrm{Si}}$, of the silicon and the atomic volumes of the hydrogen, $V_{\mathrm{H}}$, and the Ge atoms, $V_{\mathrm{Ge}}$, respectively, while $u(\mathbf{r}), v(\mathbf{r})$ are the number densities of the $\mathrm{H}$ and $\mathrm{Ge}$ atoms, respectively. The atomic scattering factor of $\mathrm{H}$ (one electron), $f_{\mathrm{H}}(E) \approx$ const, is nearly energy independent, while the atomic scattering factor of the Ge atoms, $f_{\mathrm{Ge}}(E)=f_{0, \mathrm{Ge}}+f_{\mathrm{Ge}}^{\prime}(E)+i f_{\mathrm{Ge}}^{\prime \prime}(E)$, shows strong variation with the energy in the vicinity of the K-absorption edge of Ge due to the so-called anomalous dispersion corrections $f_{\mathrm{Ge}}^{\prime}(E), f_{\mathrm{Ge}}^{\prime \prime}(E)$. Calculating the scattering intensity $I(\mathbf{q})$ $=|A(\mathbf{q})|^{2}=A(\mathbf{q}) A^{*}(\mathbf{q})$ by means of Eqs. (1) and (2) and averaging over all orientations yields a sum of three contributions $I(q, E)=\left|A_{\mathrm{H}}(q)\right|^{2}+\left|A_{\mathrm{HGe}}(q, E)\right|^{2}+\left|A_{\mathrm{Ge}}(q, E)\right|^{2}$, with the integrals: ${ }^{10}$

$$
\begin{aligned}
S_{\mathrm{H}}(q)=\left|A_{\mathrm{H}}(q)\right|^{2} & =4 \pi \Delta f_{\mathrm{H}}^{2} \int_{V_{p}} \int u(\mathbf{r}) u\left(\mathbf{r}^{\prime}\right) \frac{\sin \left(q\left|\mathbf{r}-\mathbf{r}^{\prime}\right|\right.}{q\left|\mathbf{r}-\mathbf{r}^{\prime}\right|} \\
S_{\mathrm{HGe}}(q, E)= & \left|A_{\mathrm{HGe}}(q, E)\right|^{2} \\
= & 4 \pi \cdot 2 \Delta f_{\mathrm{H}}\left[f_{0, \mathrm{Ge}}-\rho_{\mathrm{Si}} V_{\mathrm{Ge}}\right. \\
& \left.+f_{\mathrm{Ge}}^{\prime}(E)\right] \int_{V_{p}} u(\mathbf{r}) v\left(\mathbf{r}^{\prime}\right) \\
& \times \frac{\sin \left(q\left|\mathbf{r}-\mathbf{r}^{\prime}\right|\right)}{q\left|\mathbf{r}-\mathbf{r}^{\prime}\right|} d^{3} r d^{3} r^{\prime}, \\
S_{\mathrm{Ge}}(q, E)= & \left|A_{\mathrm{Ge}}(q, E)\right|^{2} \\
= & 4 \pi \cdot\left|\Delta f_{\mathrm{Ge}}(E)\right|^{2} \int_{V_{p}} \int(\mathbf{r}) v\left(\mathbf{r}^{\prime}\right) \\
& \times \frac{\sin \left(q\left|\mathbf{r}-\mathbf{r}^{\prime}\right|\right)}{q\left|\mathbf{r}-\mathbf{r}^{\prime}\right|} d^{3} r d^{3} r^{\prime} .
\end{aligned}
$$$$
=4 \pi \Delta f_{\mathrm{H}}^{2} \int_{V_{p}} \int u(\mathbf{r}) u\left(\mathbf{r}^{\prime}\right) \frac{\sin \left(q\left|\mathbf{r}-\mathbf{r}^{\prime}\right|\right)}{q\left|\mathbf{r}-\mathbf{r}^{\prime}\right|} d^{3} r d^{3} r^{\prime},
$$

Equations (3) give the nonresonant scattering of the $\mathrm{H}$, $S_{\mathrm{H}}(q)$, the cross-term or mixed-resonant scattering, $S_{\mathrm{HGe}}(q, E)$, originating from the superposition of the scattering amplitudes of the $\mathrm{H}$ and $\mathrm{Ge}$ atoms and the scattering of the Ge, $S_{\mathrm{Ge}}(q, E)$, which contains the so-called pure-resonant scattering. By measuring the scattering curves $I\left(q, E_{1}\right)$, $I\left(q, E_{2}\right)$, and $I\left(q, E_{3}\right)$ at three energies in the vicinity of the $\mathrm{K}$-absorption edge of Ge the two separated scattering curves $\Delta I\left(q, E_{1}, E_{2}\right)=I\left(q, E_{1}\right)-I\left(q, E_{2}\right)$ and $\Delta I\left(q, E_{1}, E_{3}\right)=I\left(q, E_{1}\right)$ $-I\left(q, E_{3}\right)$ are obtained and from these the form factor, $S_{\mathrm{Ge}}^{\text {form }}(q)$, of the spatial distribution of the Ge component in the alloy can be calculated as described in more detail in ${ }^{11}$

$$
\begin{array}{r}
S_{\mathrm{Ge}}^{\mathrm{form}}(q)=4 \pi \int_{V_{p}} \int v(\mathbf{r}) v\left(\mathbf{r}^{\prime}\right) \frac{\sin \left(q\left|\mathbf{r}-\mathbf{r}^{\prime}\right|\right)}{q\left|\mathbf{r}-\mathbf{r}^{\prime}\right|} d^{3} r d^{3} r^{\prime} \\
=\left[\frac{\Delta I\left(q, E_{1}, E_{2}\right)}{f_{\mathrm{Ge}}^{\prime}\left(E_{1}\right)-f_{\mathrm{Ge}}^{\prime}\left(E_{2}\right)}-\frac{\Delta I\left(q, E_{1}, E_{3}\right)}{f_{\mathrm{Ge}}^{\prime}\left(E_{1}\right)-f_{\mathrm{Ge}}^{\prime}\left(E_{3}\right)}\right] \\
\cdot \frac{1}{F\left(E_{1}, E_{2}, E_{3}\right)}, \\
F\left(E_{1}, E_{2}, E_{3}\right)=f_{\mathrm{Ge}}^{\prime}\left(E_{2}\right)-f_{\mathrm{Ge}}^{\prime}\left(E_{3}\right)+\frac{f_{\mathrm{Ge}}^{\prime \prime 2}\left(E_{1}\right)-f_{\mathrm{Ge}}^{\prime \prime 2}\left(E_{2}\right)}{f_{\mathrm{Ge}}^{\prime}\left(E_{1}\right)-f_{\mathrm{Ge}}^{\prime}\left(E_{2}\right)} \\
-\frac{f_{\mathrm{Ge}}^{\prime \prime 2}\left(E_{1}\right)-f_{\mathrm{Ge}}^{\prime \prime 2}\left(E_{3}\right)}{f_{\mathrm{Ge}}^{\prime}\left(E_{1}\right)-f_{\mathrm{Ge}}^{\prime}\left(E_{3}\right)} .
\end{array}
$$

With Eq. (4) ASAXS provides a technique to access directly the scattering of the Ge component and structural information on the Ge distribution in the alloy can be obtained from the analysis of the form factor $S_{\mathrm{Ge}}^{\text {form }}(q)$. More generally, Eq. (4) provides a method to access directly, by analytical means, the pure-resonant scattering contribution by measuring the small-angle scattering at only three suitable energies. From $S_{\mathrm{Ge}}^{\text {form }}(q)$ the three basic scattering functions of Eq. (3) can be calculated as is shown for the energies $E_{1}$ and $E_{2}$ as follows:

$$
\begin{aligned}
& S_{\mathrm{Ge}}\left(q, E_{1}\right)=\left|\Delta f_{\mathrm{Ge}}\left(E_{1}\right)\right|^{2} \cdot S_{\mathrm{Ge}}^{\mathrm{form}}(q), \\
& S_{\mathrm{HGe}}\left(q, E_{1}\right)= {\left[\Delta I\left(q, E_{1}, E_{2}\right)-S_{\mathrm{Ge}}\left(q, E_{1}\right)\right.} \\
&\left.+S_{\mathrm{Ge}}\left(q, E_{2}\right)\right] \frac{\left(f_{0, \mathrm{Ge}}-\rho_{\mathrm{Si}} V_{\mathrm{Ge}}\right)+f_{\mathrm{Ge}}^{\prime}\left(E_{1}\right)}{f_{\mathrm{Ge}}^{\prime}\left(E_{1}\right)-f_{\mathrm{Ge}}^{\prime}\left(E_{2}\right)}, \\
& S_{\mathrm{H}}(q)=I\left(q, E_{1}\right)-S_{\mathrm{HGe}}\left(q, E_{1}\right)-S_{\mathrm{Ge}}\left(q, E_{1}\right) .
\end{aligned}
$$

In addition to the structural information obtained from the three basic functions, important quantitatve information related to the amount of $\mathrm{H}$ and Ge localized in different types of inhomogeneities can be deduced from the integrals of the basic scattering functions. The integral of the total scattering curve $I(q, E)$ (the so-called invariant $\left.{ }^{12}\right)$ can be correlated to the photoconductivity as shown later.

$$
\begin{aligned}
& Q_{\mathrm{tot}}(E)=\frac{1}{4 \pi} \int_{Q} I(q, E) d^{3} q, \\
& Q_{\mathrm{Ge}}(E)=\frac{1}{4 \pi} \int_{Q} S_{\mathrm{Ge}}(q, E) d^{3} q, \\
& Q_{\mathrm{H}}=\frac{1}{4 \pi} \int_{Q} S_{\mathrm{H}}(q) d^{3} q .
\end{aligned}
$$

\section{EXPERIMENT}

Two series of HWCVD films $(0.60<x<0.70)$ were made at NREL applying different filament and substrate temperatures, $T_{f}$ and $T_{s}$, respectively. The deposition conditions are described elsewhere along with some optoelectronic properties of the first series of such alloys made by the HWCVD method. ${ }^{6}$ For ASAXS, films were deposited on high purity, 10- $\mu \mathrm{m}$-thick Al foils, which were then folded 
TABLE I. Amorphous silicon-germanium alloys $a-\mathrm{Si}_{x} \mathrm{Ge}_{1-x}: \mathrm{H}$ prepared by the HWCVD technique at different filament and substrate temperatures $T_{f}$ and $T_{S}$. $C_{\mathrm{H}}$ is the hydrogen content and $E_{\text {Tauc }}$ the materials band gap and $d$ the thickness of the films. In the eighth column the calculated atomic number densities of Ge in the alloys are listed assuming a mass density of $96 \%$ that of $c-\mathrm{Si}_{1-x} \mathrm{Ge}_{x}$. The last two columns give the photoconductivities (PC) and the deposition rates (DR), respectively.

\begin{tabular}{|c|c|c|c|c|c|c|c|c|c|}
\hline $\begin{array}{c}\text { Sample } \\
\text { series }\end{array}$ & $\begin{array}{c}T_{f} \\
\left({ }^{\circ} \mathrm{C}\right)\end{array}$ & $\begin{array}{c}T_{S} \\
\left({ }^{\circ} \mathrm{C}\right)\end{array}$ & $x$ & $\begin{array}{c}C_{\mathrm{H}} \\
\text { (at } \% \text { ) }\end{array}$ & $\begin{array}{c}E_{\text {Tauc }} \\
(\mathrm{eV})\end{array}$ & $\begin{array}{c}d \\
(\mu \mathrm{m})\end{array}$ & $\begin{array}{c}n_{\mathrm{Ge}} \\
10^{22} \\
\left(\mathrm{~cm}^{-3}\right)\end{array}$ & $\begin{array}{c}\mathrm{PC} \\
10^{-6} \\
(\mathrm{Ohm} \mathrm{cm})^{-1}\end{array}$ & $\begin{array}{c}\mathrm{DR} \\
(\mathrm{nm} / \mathrm{sec})\end{array}$ \\
\hline$L 990$ & 2150 & 180 & 0.62 & 15.0 & 1.30 & 0.53 & 2.611 & 0.239 & 0.89 \\
\hline L991 & 2065 & 180 & 0.63 & 15.2 & 1.29 & 0.51 & 2.653 & 0.28 & 0.75 \\
\hline L992 & 1975 & 180 & 0.64 & 15.5 & 1.28 & 0.54 & 2.695 & 0.356 & 0.64 \\
\hline$L 993$ & 1885 & 180 & 0.64 & 15.8 & 1.28 & 0.44 & 2.695 & 0.751 & 0.46 \\
\hline L994 & 1800 & 180 & 0.67 & 14.5 & 1.25 & 0.33 & 2.821 & 6.11 & 0.27 \\
\hline L995 & 1750 & 180 & 0.70 & 12.5 & 1.22 & 0.32 & 2.948 & 5.11 & 0.14 \\
\hline \multicolumn{10}{|l|}{ Series } \\
\hline L998 & 1800 & 360 & 0.60 & 3.0 & 1.21 & 0.45 & 2.527 & 0.353 & 0.37 \\
\hline L999 & 1800 & 305 & 0.63 & 5.0 & 1.24 & 0.42 & 2.653 & 0.898 & 0.32 \\
\hline$L 1000$ & 1800 & 260 & 0.64 & 9.5 & 1.27 & 0.48 & 2.695 & 1.95 & 0.33 \\
\hline$L 1001$ & 1800 & 210 & 0.66 & 12.0 & 1.29 & 0.49 & 2.779 & 2.10 & 0.33 \\
\hline$L 1002$ & 1800 & 159 & 0.68 & 19.0 & 1.31 & 0.49 & 2.864 & 0.825 & 0.33 \\
\hline$L 1003$ & 1800 & 130 & 0.69 & 21.0 & 1.32 & 0.48 & 2.906 & 0.965 & 0.32 \\
\hline
\end{tabular}

into four layers (one into eight layers) for the ASAXS measurements. Table I summarizes deposition parameters, film thickness, and optoelectronic parameters of the samples under investigation.

The ASAXS measurements were carried out at the JUSIFA beamline at HASYLAB, DESY Hamburg. ${ }^{13}$ Measurements were made for each sample with a twodimensional detector at four energies. A $q$ range from about 0.06-7 $\mathrm{nm}^{-1}$ (fix exponent-note) was covered. Table II provides the anomalous dispersion corrections for $\mathrm{Si}$ and $\mathrm{Ge}$ at the four energies used here based on the calculations of Cromer and Liberman. ${ }^{14,15}$ These were used to permit the separation of the Ge-related pure-resonant scattering described in detail by Eqs. (1)-(5). From the four energies different combinations, $E_{1}, E_{2}$, and $E_{3}$ and $E_{1}, E_{2}$, and $E_{4}$, were used to calculate the form factor of the spatial Ge distribuiton in the alloy. Constant background effects due to diffuse scattering ${ }^{1}$ and due to resonant Raman scattering occuring near the K-absorption edge at $11.103 \mathrm{keV}$ have been subtracted from the measured intensities. The scattering intensity is calibrated into macroscopic scattering cross section in units of cross section per unit volume $\left(\mathrm{cm}^{2} / \mathrm{cm}^{3}\right)$ $=\left(\mathrm{cm}^{-1}\right)$.

\section{RESULTS}

Figures 1(a) and 1(b) show the total (a) and separated (b) scattering curves of the series 1 of HWCVD-alloys prepared

TABLE II. Anomalous dispersion corrections of Si and Ge (Refs. 14 and $15)$.

\begin{tabular}{|c|c|c|c|c|c|}
\hline No. & $\begin{array}{c}E \\
(\mathrm{keV})\end{array}$ & $f_{\mathrm{Ge}}^{\prime}$ & $f_{\mathrm{Ge}}^{\prime \prime}$ & $f_{\mathrm{Si}}^{\prime}$ & $f_{\mathrm{Si}}^{\prime \prime}$ \\
\hline 1 & 10.053 & -2.093 & 0.592 & 0.181 & 0.215 \\
\hline 2 & 10.996 & -4.005 & 0.504 & 0.158 & 0.180 \\
\hline 3 & 11.083 & -5.996 & 0.495 & 0.155 & 0.176 \\
\hline 4 & 11.098 & -7.458 & 0.493 & 0.155 & 0.176 \\
\hline
\end{tabular}

at different $T_{f}$ between 1750 and $2150{ }^{\circ} \mathrm{C}$ and a fixed $T_{s}$ of $180^{\circ} \mathrm{C}$. The separated scattering curves were obtained by subtracting the total scattering curves measured at the energies 11083 and $10053 \mathrm{eV}$. The SAXS curves strongly depend on the filament temperature especially in the $q$ range between 0.2 and $5 \mathrm{~nm}^{-1}$. The integrated SAXS $Q_{\text {tot }}$ [invariant-Eq. (6)] shows a monotonic increase with the filament temperature (Fig. 2 left ordinate) and is correlated with a strong decrease of the photoconductivity of the films (Fig. 2 right ordinate). No pure-resonant scattering contribution could be obtained from this series. When calculating the $S_{\mathrm{Ge}}^{\text {form }}(q)$ of the pure-resonant scattering contribution [Eq. (4)] the data points scatter along the abscissa indicating little or no pure-resonant contribution (probably less than 0.001 of the total scattering).

The second series (variable $T_{s}$ ) shows a completely different behavior. Figures 3-5 show the scattering curves of three samples from the second series prepared at a fixed $T_{f}$ and the three substrate temperatures, $T_{s}=360,305$, and $210{ }^{\circ} \mathrm{C}$. The circles represent the total scattering curves measured at $10053 \mathrm{eV}$, while the squares show the separated scattering curves (mixed resonant) obtained from SAXS measurements at the two energies 10053 and $11083 \mathrm{eV}$. When calculating the $S_{\mathrm{Ge}}^{\mathrm{form}}(q)$ of the spatial distribution of Ge [Eq. (4)] for all six samples, significant scattering cross sections were obtained (triangles). The integrated form factor amounts to between $0.4 \%$ and $5 \%$ of the integrated total scattering intensity (last column in Table III). From the different combinations of the energies $E_{1}, E_{2}$, and $E_{3}$ and $E_{1}$, $E_{2}$, and $E_{4}$, form factors with the same shape within the error bars were obtained and for the further calculations the averaged form factor of the two calculations was employed. The triangles in Figs. 3-5 represent the form factors of three samples and the solid lines passing through the data points are fitted model functions according to the following $q$ dependence: 

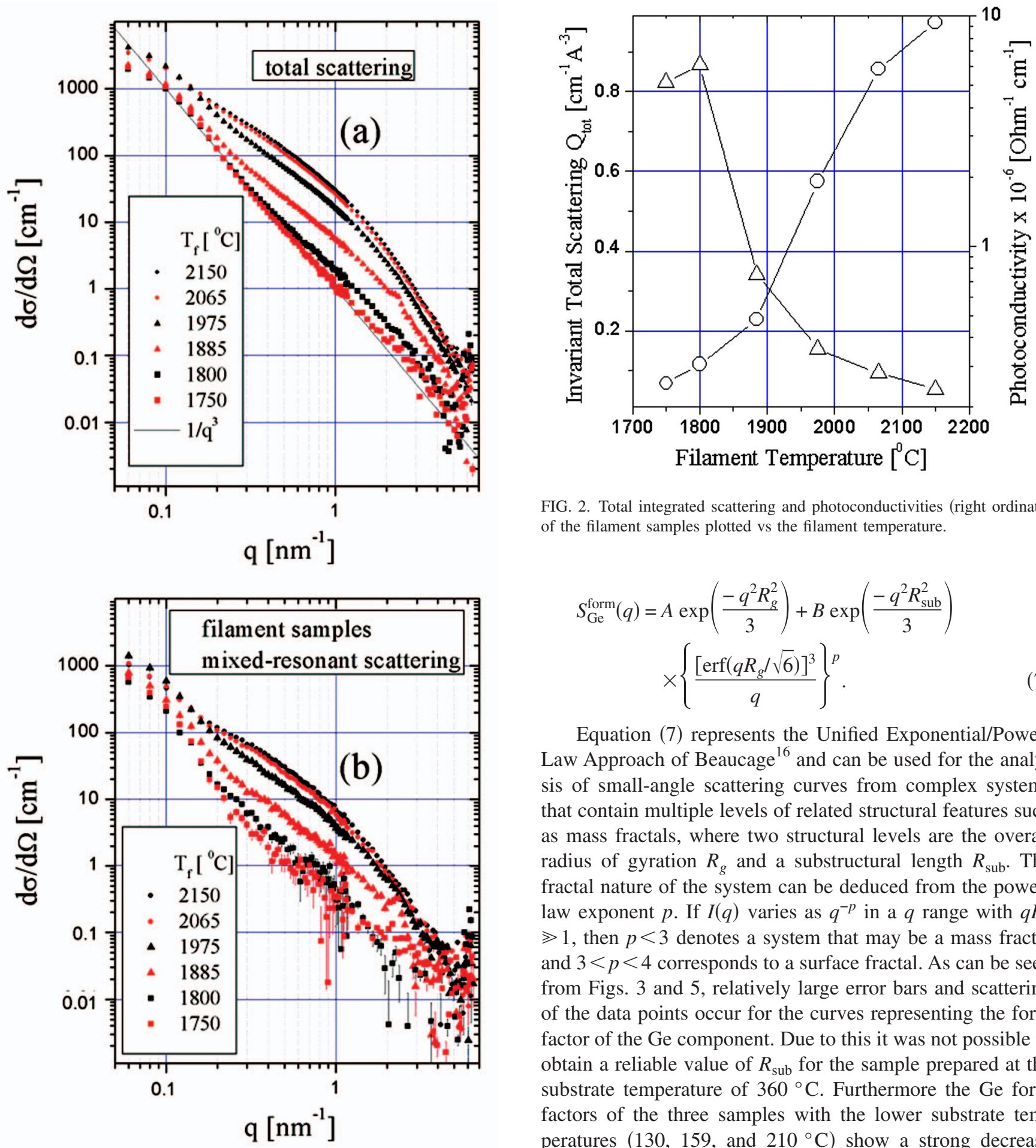

FIG. 2. Total integrated scattering and photoconductivities (right ordinate) of the filament samples plotted vs the filament temperature.

$$
\begin{aligned}
S_{\mathrm{Ge}}^{\mathrm{form}}(q)= & A \exp \left(\frac{-q^{2} R_{g}^{2}}{3}\right)+B \exp \left(\frac{-q^{2} R_{\mathrm{sub}}^{2}}{3}\right) \\
& \times\left\{\frac{\left[\operatorname{erf}\left(q R_{g} / \sqrt{6}\right)\right]^{3}}{q}\right\}^{p} .
\end{aligned}
$$

Equation (7) represents the Unified Exponential/PowerLaw Approach of Beaucage ${ }^{16}$ and can be used for the analysis of small-angle scattering curves from complex systems that contain multiple levels of related structural features such as mass fractals, where two structural levels are the overall radius of gyration $R_{g}$ and a substructural length $R_{\text {sub. The }}$ fractal nature of the system can be deduced from the powerlaw exponent $p$. If $I(q)$ varies as $q^{-p}$ in a $q$ range with $q R_{g}$ $\gg 1$, then $p<3$ denotes a system that may be a mass fractal and $3<p<4$ corresponds to a surface fractal. As can be seen from Figs. 3 and 5, relatively large error bars and scattering of the data points occur for the curves representing the form factor of the Ge component. Due to this it was not possible to obtain a reliable value of $R_{\text {sub }}$ for the sample prepared at the substrate temperature of $360{ }^{\circ} \mathrm{C}$. Furthermore the Ge form factors of the three samples with the lower substrate temperatures $\left(130,159\right.$, and $\left.210^{\circ} \mathrm{C}\right)$ show a strong decrease with the result of statistically noisy data (Fig. 5). Especially at $q$ values beyond $3 \mathrm{~nm}^{-1}$ the data points scatter around zero causing strong variations of the fitted power-law exponent. This is demonstrated in Fig. 5 by the two solid lines, which represent different fits of the model function, when the fitting procedure is restricted to different $q$ intervals. From the form factors of these three samples no reliable structure information concerning the power-law exponent could be obtained. Table III summarizes the results for the samples of the second series obtained from the fitting procedure. Beaucage's model is used in the following course of the paper for a quantitative description of suggested fractal structures, because other models such as a bimodal size distribution of 


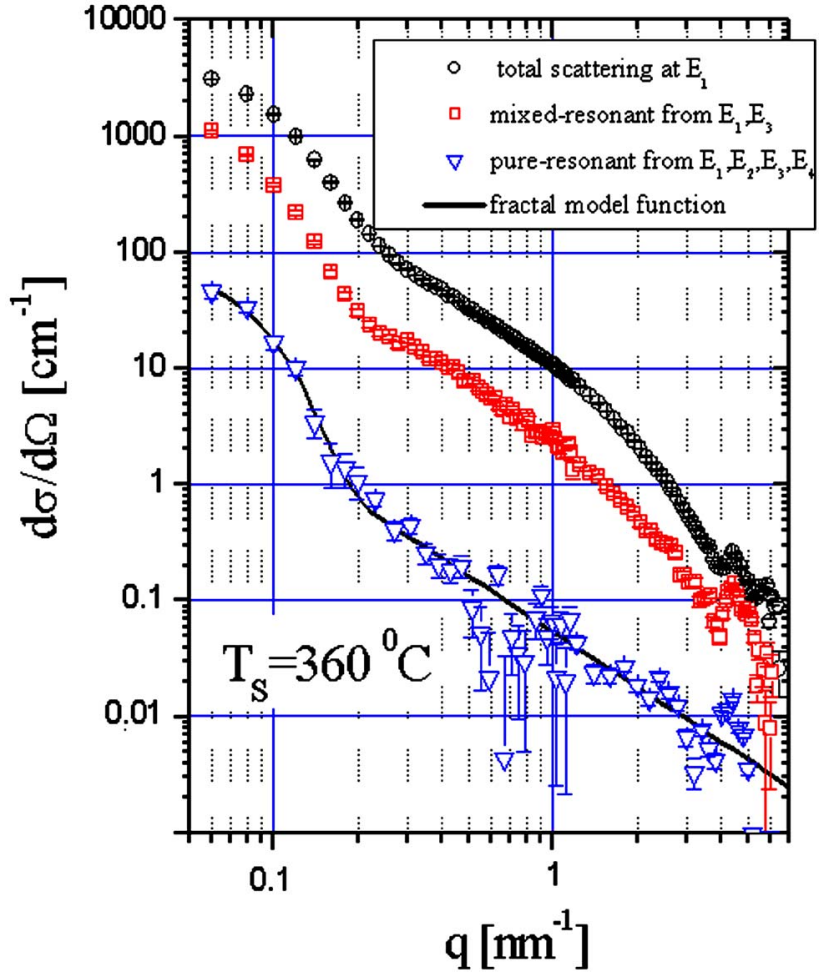

FIG. 3. Total scattering, separated scattering and the form factor of the pure-resonant scattering contribution of one sample from the second series prepared at the substrate temperature of $360^{\circ} \mathrm{C}$. The solid line represents the fractal model function. The origin of the peaklike structure at $4.5 \mathrm{~nm}^{-1}$ in the total scattering curve is unknown.

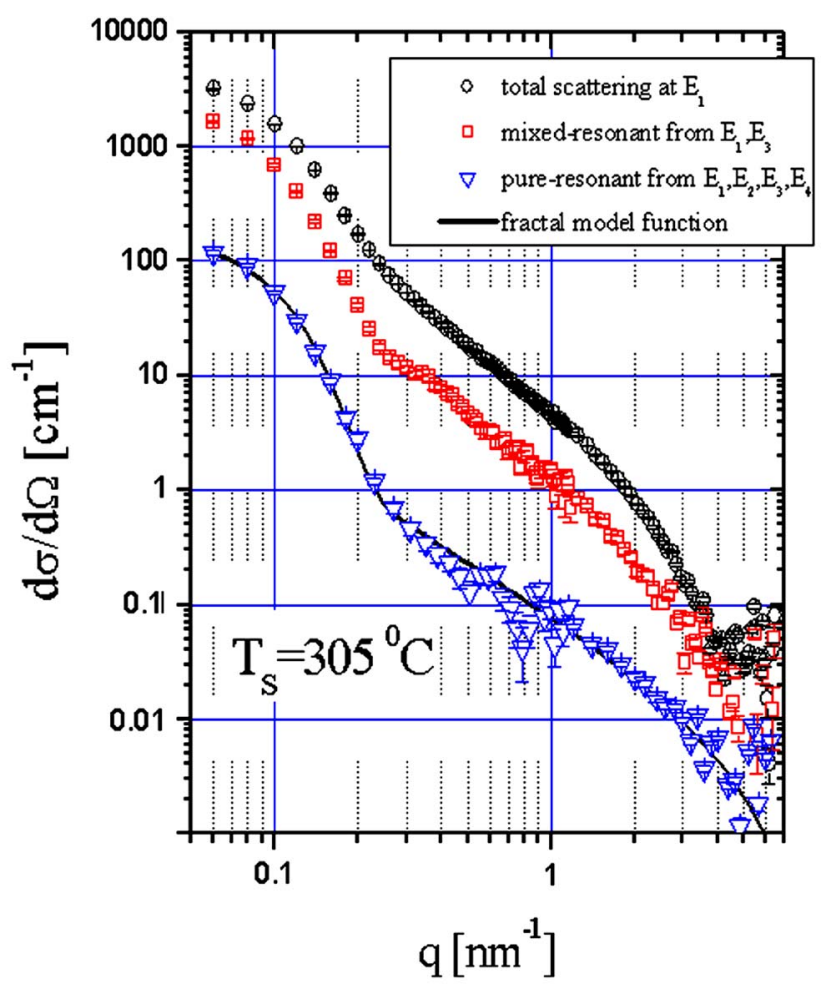

FIG. 4. Total scattering, separated scattering, and the form factor of the pure-resonant scattering contribution of one sample from the second series prepared at the substrate temperature of $305^{\circ} \mathrm{C}$. The solid line represents the fractal model function.

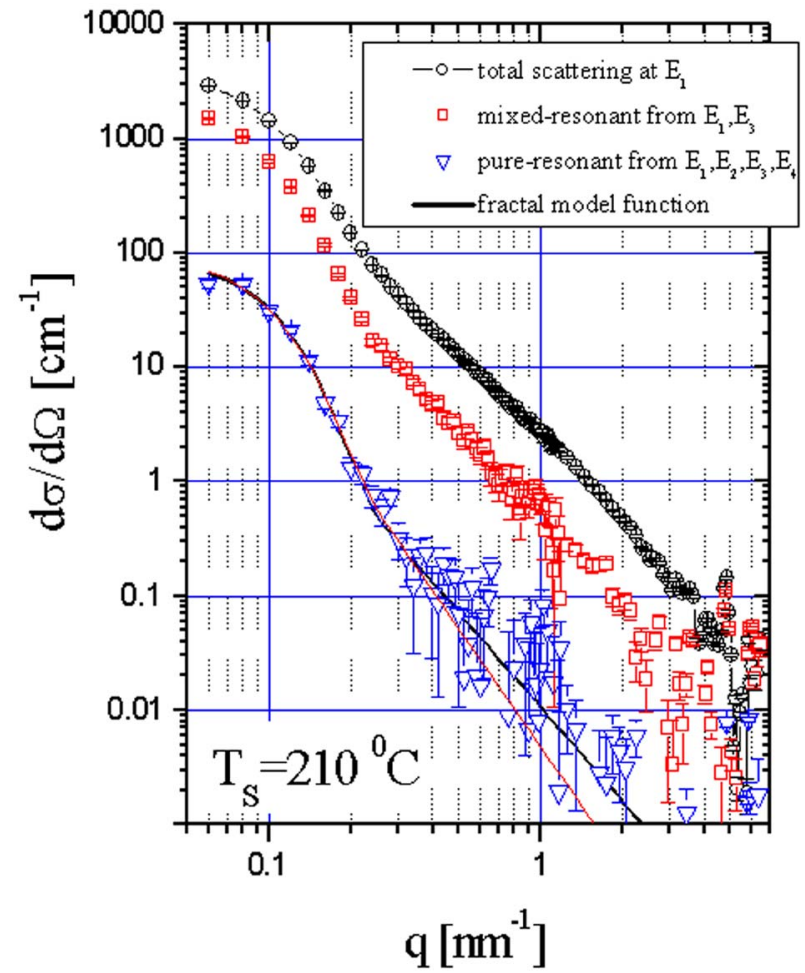

FIG. 5. Total scattering, separated scattering, and the form factor of the pure-resonant scattering contribution of one sample from the second series prepared at the substrate temperature of $210^{\circ} \mathrm{C}$. The solid line represents the fractal model function.

spherically shaped clusters or a size distribution of cylinders failed, when employed to fit the form factors of the Ge component. Definitive proof of fractal structures in these alloys will require further research.

\section{DISCUSSION}

As mentioned in the previous chapter the data points scatter around zero when calculating the form factor, $S_{\mathrm{Ge}}^{\text {form }}(q)$, of the $T_{f}$ series indicating that the variation of the scattering curves due to the pure-resonant scattering contribution is so small it cannot be resolved. On the other hand the separated scattering curves [Fig. 1(b)] from two energies show clearly that the Ge must be inhomogeneously distributed in the alloy. Additionally one can note that the separated

TABLE III. Fit results of the six substrate alloys based on Eq. (6). The fitted scattering curves of the alloys with $T_{S}=360,305,210{ }^{\circ} \mathrm{C}$ are plotted in Figs. $3-5$. The parameters with quotation mark indicate the fits to the form factors with poor or no statistical significance at the higher $q$ values. The last column gives the amount of the integrated form factor to the integrated total scattering.

\begin{tabular}{ccccccc}
\hline \hline $\begin{array}{c}T_{S} \\
\left({ }^{\circ} \mathrm{C}\right)\end{array}$ & $\begin{array}{c}A \\
\left(\mathrm{~cm}^{-1}\right)\end{array}$ & $\begin{array}{c}R_{g} \\
(\mathrm{~nm})\end{array}$ & $\begin{array}{c}R_{\text {sub }} \\
(\mathrm{nm})\end{array}$ & $\begin{array}{c}\mathrm{B} \times 10^{-4} \\
\left(\mathrm{~cm}^{-1}\right)\end{array}$ & $p$ & $\begin{array}{c}Q_{\mathrm{Ge}}^{\text {form }} / Q_{\text {tot }} \\
(\%)\end{array}$ \\
\hline 360 & 88.3 & 22.3 & $\ldots$ & 14.26 & 1.58 & 1.07 \\
305 & 185.9 & 19.3 & 0.4 & 21.91 & 1.56 & 3.92 \\
260 & 177.6 & 18.9 & 0.3 & 20.03 & 1.37 & 4.87 \\
210 & 106.0 & 19.2 & $\cdots$ & $0.02(?)$ & $3.4(?)$ & 0.41 \\
159 & 316.7 & 18.9 & $\ldots$ & $0.18(?)$ & $2.8(?)$ & 0.68 \\
130 & 119.5 & 21.0 & $\ldots$ & $1.08(?)$ & $1.9(?)$ & 0.75 \\
\hline \hline
\end{tabular}


scattering curves have similar or identical shape compared to the related total scattering curves. The most probable explanation for this behavior is the presence of voids or a twophase mixture in the alloys especially at the temperatures beyond $T_{f}=1800{ }^{\circ} \mathrm{C}$ as discussed in an earlier paper. ${ }^{3}$ It is noteworthy that the asymptotic behavior of the scattering curves change from a $q^{-4}$ behavior (smooth interfaces) to a different power law with an exponent smaller than 4 at the two lower filament temperatures, where the invariant is diminished by an order of magnitude. Due to the minimum of the integrated intensity at the lowest filament temperature the film reaches a minimum of inhomogeneity at $1750{ }^{\circ} \mathrm{C}$.

The scattering curves of the second sample series show a significantly different behavior. Again the scattering curves strongly depend on the preparation parameters, here the variation of the substrate temperature. But in contrast to the $T_{f}$ series the form factor, $S_{\mathrm{Ge}}^{\text {form }}(q)$, can be extracted for all samples of the $T_{s}$ series. The shape of the separated (mixed resonant) and the pure-resonant (Ge form factor) scattering curves reveal significant differences indicating the presence of a third phase probably voids or hydrogen clusters. From three phases-for example, a region in the alloy with Si containing only a small amount of $\mathrm{Ge}$ and $\mathrm{H}$, a second region with a higher amount of $\mathrm{Ge}$ and additional $\mathrm{H}$, and finally voids with only small amount of $\mathrm{H}$ - the two difference electron densities $\Delta \rho_{\mathrm{H}}, \Delta \rho_{\mathrm{Ge}}$ of Eq. (2) originate. Because the functions $u(\mathbf{r}), v(\mathbf{r})$, which describe the spatial distributions of $\mathrm{H}$ and $\mathrm{Ge}$ atoms, respectively, are different, the shape of the separated scattering curve changes with the energy, because it is composed of the two contributions, $S_{\mathrm{HGe}}(q, E)$, $S_{\mathrm{Ge}}(q, E)$, which contribute differently at different energies. Due to this the separated scattering curves and the form factors of Figs. 3-5 show different shape. In the case of a twophase mixture (only one contrast) the functions, $u(\mathbf{r}), v(\mathbf{r})$, are linearly dependent and the basic functions of Eq. (3) simply scale.

Figure 6(a) shows on the left ordinate the integrated intensity (invariant) of the $T_{s}$ series with a minimum at $260{ }^{\circ} \mathrm{C}$. For comparison the integrated intensity of the pureresonant scattering, which was calculated from the Ge form factor [Eqs. (5)], divided by the invariant $Q_{\mathrm{Ge}} / Q_{\mathrm{tot}}$ [Eqs. (6)] is plotted on the right ordinate. While the invariant reaches a minimum the ratio of integrated pure-resonant and total scattering reaches a maximum at the same $T_{s}=260{ }^{\circ} \mathrm{C}$. The mixed-resonant and the nonresonant scattering contributions were calculated from Eqs. (5). The integrals of Eqs. (3) define a symmetric, positive definite bilinear form in the space of functions. Due to this the Cauchy-Schwarz-Inequation must be valid $S_{\mathrm{HGe}}^{2} \leqslant 4 \cdot S_{\mathrm{Ge}} \cdot S_{\mathrm{H}}$. The basic functions calculated from Eqs. (5) fulfill this inequation within the error bars. From these scattering curves the integrated scattering $Q_{\mathrm{HGe}}, Q_{\mathrm{H}}$ and subsequently the ratios $Q_{\mathrm{HGe}} / Q_{\mathrm{tot}}$ and $Q_{\mathrm{H}} / Q_{\mathrm{tot}}$ were calculated. The results are shown in Fig. 6(b). The sign of the mixed-resonant scattering contribution [right ordinate of Fig. 6(b)] is negative due to the negative sign of the electron density difference caused by the hydrogen atoms in the amorphous Si matrix [Eqs. (3) and (5)]. The absolute units of the three scattering contributions can be obtained by multiplying the normalized values with the related values of the
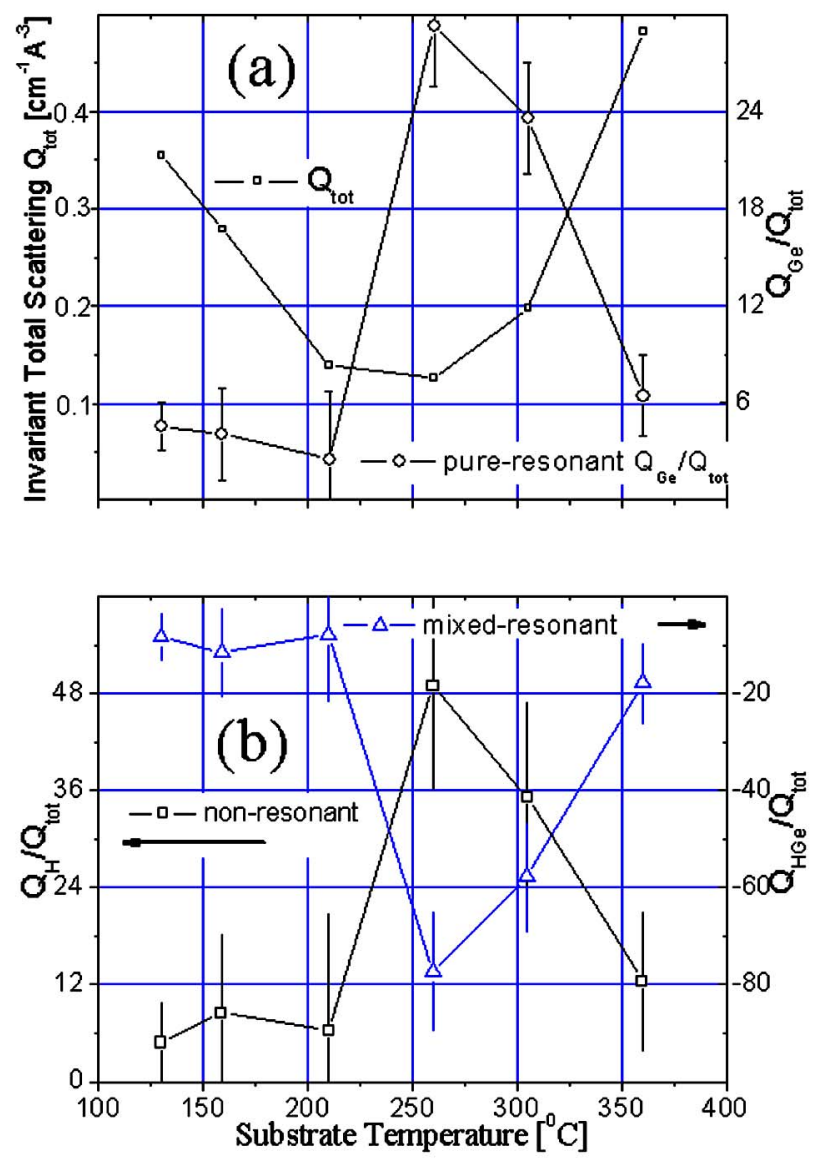

FIG. 6. The integrated intensities (invariant) of: (a) the total scattering $Q_{\text {tot }}$ (left ordinate), (a) the normalized pure-resonant scattering $Q_{\mathrm{Ge}} / Q_{\mathrm{tot}}$ (right), (b) the normalized nonresonant scattering $Q_{\mathrm{H}} / Q_{\text {tot }}$ (left) and (b) the normalized mixed-resonant scattering $Q_{\mathrm{HGe}} / Q_{\text {tot }}$ (right). Note the negative sign of the mixed-resonant contribution. The absolute units of $Q_{\mathrm{Ge}}, Q_{\mathrm{HGe}}$, and $Q_{\mathrm{H}}$ can be obtained by multiplying with the related total scattering in Fig. 6(a).

invariant, which can be read on the left ordinate of Fig. 6(a). From Figs. 6(a) and 6(b) it is obvious that the total smallangle scattering reaches a minimum while the ratio of integrated pure-resonant and total scattering $Q_{\mathrm{Ge}} / Q_{\text {tot }}$ reaches a maximum at $260^{\circ} \mathrm{C}$, i.e., the formation of Ge-related (fractal) structures reaches a relative maximum while the degree of total inhomogenity in the film is declining as $T_{s}$ approaches this intermediate substrate temperature.

This can be expressed more quantitatively. Because voids contribute to the scattering nearly in the same way as $\mathrm{H}$ (Ref. 17) the following argumentation will hold for hydrogen clusters and voids. The integrated intensities of Eqs. $(6)^{12}$ are

$$
\begin{aligned}
& Q_{\mathrm{Ge}}(E)=(2 \pi)^{3} r_{0}^{2} \cdot \Delta f_{\mathrm{Ge}}^{2}(E) \Delta n_{\mathrm{Ge}}^{2} \cdot \Phi_{\mathrm{Ge}}\left(1-\Phi_{\mathrm{Ge}}\right), \\
& Q_{\mathrm{H}}=(2 \pi)^{3} r_{0}^{2} \cdot \Delta f_{\mathrm{H}}^{2} \cdot \Delta n_{\mathrm{H}}^{2} \cdot \Phi_{\mathrm{H}}\left(1-\Phi_{\mathrm{H}}\right),
\end{aligned}
$$

where $\Delta n_{\mathrm{Ge}}, \Delta n_{\mathrm{H}}$ are the excess atomic number densities of Ge and hydrogen (and/or voids) with respect to the surrounding matrix and $\Phi_{\mathrm{Ge}}, \Phi_{\mathrm{H}}$ are the volume fractions of the inhomogeneities caused by Ge and hydrogen clusters (and/or voids), respectively. $r_{0}=2.82 \times 10^{-13} \mathrm{~cm}$ is the classical electron radius. From the integrated intensities $Q_{\mathrm{Ge}}, Q_{\mathrm{H}}$ the quantities $n_{\mathrm{Ge}}^{f}, n_{\mathrm{H}}^{f}$ can be calculated, where the index $f$ indicates a 

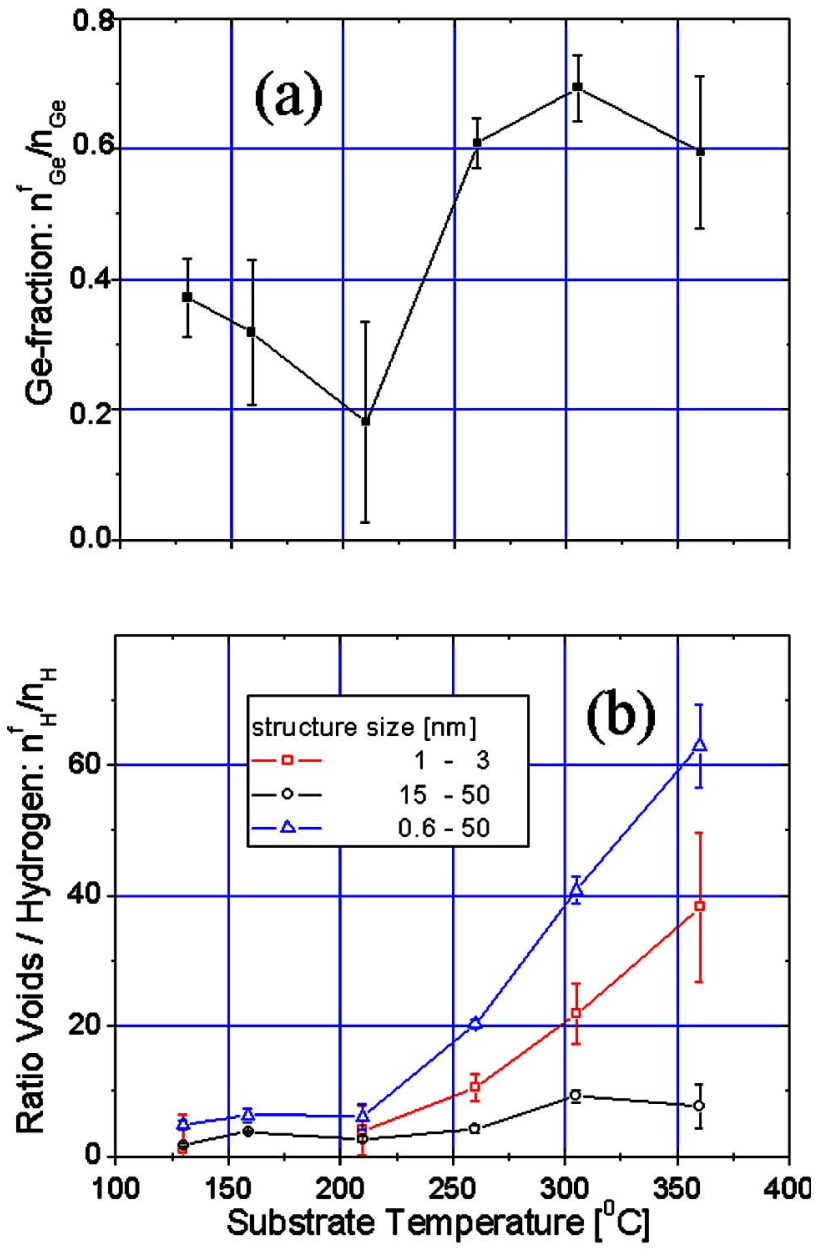

FIG. 7. The fraction of Ge atoms implemented in the suggested fractal structure (a). The ratio of void scattering relative to the scattering expected from the hydrogen implemented in the amorphous matrix (b).

fraction of $\mathrm{Ge}$ and $\mathrm{H}$ atoms (and/or voids), respectively

$$
\begin{aligned}
& n_{\mathrm{Ge}}^{f}=\Delta n_{\mathrm{Ge}} \sqrt{\Phi_{\mathrm{Ge}}\left(1-\Phi_{\mathrm{Ge}}\right)}=\sqrt{\frac{Q_{\mathrm{Ge}}(E)}{(2 \pi)^{3} r_{0}^{2} \cdot \Delta f_{\mathrm{Ge}}^{2}(E)}}, \\
& n_{\mathrm{H}}^{f}=\Delta n_{\mathrm{H}} \sqrt{\Phi_{\mathrm{H}}\left(1-\Phi_{\mathrm{H}}\right)}=\sqrt{\frac{Q_{\mathrm{H}}}{(2 \pi)^{3} r_{0}^{2} \cdot \Delta f_{\mathrm{H}}^{2}}} .
\end{aligned}
$$

The product between the equal signs is composed from the contrast between the atomic number densities in the inhomogeneities and the surrounding matrix and the volume fractions of inhomogeneities caused by $\mathrm{Ge}$ and $\mathrm{H}$ (and/or voids), respectively. For the determination of both-contrast and volume fraction-SAXS measurements at more than three or four energies are neccessary as was outlined in a previous publication. ${ }^{3}$ This is beyond the scope of the present investigation but nevertheless the quantities $n_{\mathrm{Ge}}^{f}, n_{\mathrm{H}}^{f}$ give the number densities of $\mathrm{Ge}$ and $\mathrm{H}$ atoms (and/or voids) in the sample, which contribute to the small-angle scattering, i.e., $n_{\mathrm{Ge}}^{f}, n_{\mathrm{H}}^{f}$ give the numbers of $\mathrm{Ge}$ and $\mathrm{H}$ atoms (and/or voids) which are inhomogeneously distributed.

Figure 7(a) shows $n_{\mathrm{Ge}}^{f}$ normalized to the atomic number density of Ge atoms in the sample $n_{\mathrm{Ge}}$ (eighth column of Table I) plotted versus the substrate temperature. In this dia- gram two groups of samples can be distinguished, which correspond to the sharp decrease in Fig. 7(a) at a substrate temperature below $260^{\circ} \mathrm{C}$. At $T_{s}=260{ }^{\circ} \mathrm{C}$ and beyond, more than $60 \%$ of the Ge atoms are localized in the fractal structures, while at the lower $T_{s}$ the amount of Ge atoms incorporated into the fractals is strongly reduced. This can be due to the reduction of the volume fraction (less fractals) or due to the reduction of the contrast between the fractals and the surrounding matrix by reducing the number of $\mathrm{Ge}$ atoms in the fractals, or due to both. As a consequence a larger amount of $\mathrm{Ge}$ atoms remains homogeneously distributed in the matrix for the lower substrate temperatures.

Finally, it should be possible to gain structural and quantitative information about the hydrogen clusters or voids from the analysis of the nonresonant scattering contribution and the quantity $n_{\mathrm{H}}^{f}$. Again, it is not possible to distinguish between the contrast of the atomic number densities and the volume fraction filled by hydrogen or voids, but the normalization to the hydrogen concentration in the alloys can give interesting information as is shown in Fig. 7(b). The triangles in Fig. 7(b) represent $n_{\mathrm{H}}^{f} / n_{\mathrm{H}}$, which is the quantity $n_{\mathrm{H}}^{f}$ of Eq. (9) normalized to the hydrogen concentration $n_{\mathrm{H}}$, which was calculated from the fifth column in Table I. Especially for the temperatures beyond $250{ }^{\circ} \mathrm{C}$ the ratio shows values which are far too high, i.e., 20, 40, and 60 times higher than expected from the hydrogen concentration. From this the conclusion can be drawn that the nonresonant scattering represents not only the scattering of hydrogen-filled inhomogeneities but also gives evidence for the existence of voids, which dominate the nonresonant scattering contribution. When the integral intensity of the nonresonant contribution is calculated in the $q$ range between 1 and $3 \mathrm{~nm}^{-1}$ (corresponding to structure sizes between 3 and $1 \mathrm{~nm}$ ) the integral value reaches more than $50 \%$ of the value obtained from the overall integration, while the integration in the $q$ range between 0.06 and $0.2 \mathrm{~nm}^{-1}$ (corresponding to structure sizes between 50 and $15 \mathrm{~nm}$ ) shows only weak dependence on the temperature with clearly lower values of $n_{\mathrm{H}}^{f} / n_{\mathrm{H}}$. So one can conclude that the ratio $n_{\mathrm{H}}^{f} / n_{\mathrm{H}}$ represents the ratio of voids to hydrogen atoms (i.e., the volume fractions) and that the nonresonant scattering contribution is clearly dominated by the scattering of voids with sizes of about several nanometers at higher substrate temperatures. These voids are strongly reduced when the substrate temperature is reduced and the optimum is reached, when the formation of the Ge mass fractals reaches a relative maximum in coincidence with a strongly reduced void fraction.

This can be demonstrated by the correlation of the integrated total scattering $Q_{\text {tot }}$ with the measured values of the photoconductivity of the $T_{s}$ series shown in Fig. 8. The photoconductivity reaches maximum values where the integrated total intensities $Q_{\text {tot }}$ reaches minimum values at $T_{s}=210$ and $260{ }^{\circ} \mathrm{C}$. The minimum value at $260{ }^{\circ} \mathrm{C}$ is produced by the high amount of the mixed-resonant contribution $S_{\mathrm{HGe}}(q, E)$ at this temperature [Fig. 7(b) right ordinate] caused by a large value of the convolution integral in Eq. (3) with respect to the total scattering. Note that the negative sign is introduced by the negative sign of the contrast $\Delta f_{\mathrm{H}}$. The large value of the convolution integral indicates a strong overlap in space 


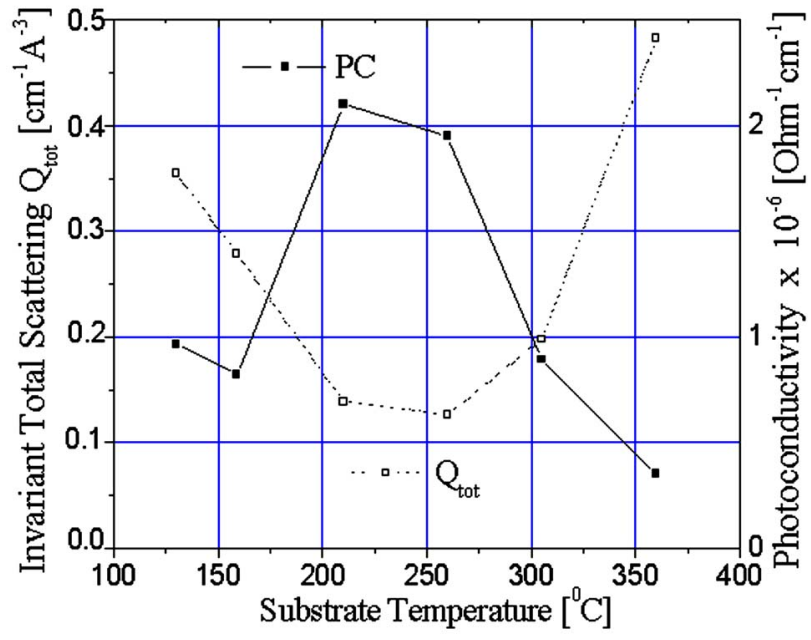

FIG. 8. Total integrated scattering and photoconductivities (right ordinate) of the substrate samples plotted vs the substrate temperature.

of the two number density functions $u(\mathbf{r}), v(\mathbf{r})$ of $\mathrm{H}$ and $\mathrm{Ge}$ atoms and from this it can be concluded that a larger amount of $\mathrm{H}$ atoms is probably bonded to $\mathrm{Ge}$ atoms. This is confirmed by IR results, which show a maximum of $\mathrm{Ge}-\mathrm{H}$ bonding fraction at $260{ }^{\circ} \mathrm{C} .{ }^{8,9}$ At $210^{\circ} \mathrm{C}$ the photoconductivity is still high though the amount of the mixed-resonant contribution is strongly reduced. Here the competing influence of the voids comes into play. Because a strong reduction of the void fraction is still going on when $T_{s}$ is reduced to $210^{\circ} \mathrm{C}$ [Fig. $7(\mathrm{~b})]$ the photoconductivity remains high though the fraction of $\mathrm{Ge}-\mathrm{H}$ bonding starts to decline (known from IR measurements ${ }^{8,9}$ ) due to the reduced number of $\mathrm{Ge}$ atoms incorporated in the fractals. For the two lowest values of $T_{s}$ 159 and $130{ }^{\circ} \mathrm{C}$ no further reduction of voids takes place and the number of $\mathrm{Ge}$ atoms incorporated in the fractals remains on a low level with the result of low photoconductivities.

\section{CONCLUSIONS}

ASAXS measurements obtained from hot wire deposited $a-\mathrm{Si}_{1-x} \mathrm{Ge}_{x}: \mathrm{H}$ alloys with $x$ between 0.6 and 0.7 reveal clear evidence of nonuniformly distributed Ge. Due to the preparation parameters strong differences of the material nanostructures were found. From the separation of the pureresonant scattering contribution, the alloys Ge component is suggested to be mainly distributed in mass fractals with the fractal dimension between 1.3 and 1.6 and sizes of about $40 \mathrm{~nm}$. More than $60 \%$ of the Ge atoms are incorporated in these structures at substrate temperatures beyond $250{ }^{\circ} \mathrm{C}$.
This amount is drastically reduced when lowering the substrate temperature. From the nonresonant scattering, evidence for the existence of voids with a size of a few nanometers was found. The scattering of the voids exceeds the scattering expected from hydrogen clusters by more than one order of magnitude. Improved material properties are reached at a substrate temperature of $260{ }^{\circ} \mathrm{C}$, where the formation of the suggested mass fractals caused by nonuniformly distributed $\mathrm{Ge}$ reaches a relative maximum in coincidence with a strongly reduced void fraction, and increased H-Ge bonding.

The effect of the $T_{f}$ reduction at fixed $T_{s}$ was to dramatically reduce the voids and Ge nonuniformity and thereby improve the photoconductivity by about a factor of 20. A further optimization by varying $T_{s}$ at fixed $T_{f}$ produced different changes in the Ge substructure indicative of enhanced Ge mass fractals, in addition to void reduction, that also improved the photoconduvtivity by about a factor of 5 .

\section{ACKNOWLEDGMENT}

The work was supported in part by the United States National Renewable Energy Laboratory under Subcontract No. XAK-8-17619-31 to the Colorado School of Mines.

${ }^{1}$ D. L. Williamson, Mater. Res. Soc. Symp. Proc. 377, 251 (1995).

${ }^{2}$ G. Goerigk and D. L. Williamson, Solid State Commun. 108, 419 (1998).

${ }^{3}$ G. Goerigk and D. L. Williamson, J. Non-Cryst. Solids 281, 181 (2001).

${ }^{4}$ G. Goerigk and D. L. Williamson, J. Appl. Phys. 90, 5808 (2001).

${ }^{5}$ E. C. Molenbroek, A. H. Mahan, and A. Gallagher, J. Appl. Phys. 82, 1909 (1997).

${ }^{6}$ B. P. Nelson et al., Mater. Res. Soc. Symp. Proc. 507, 447 (1998).

${ }^{7}$ B. P. Nelson, Y. Xu, D. L. Williamson, D. Han, R. Braunstein, M. Boshta, and B. Alavi, Thin Solid Films 430, 104 (2003).

${ }^{8}$ Y. Xu, B. P. Nelson, D. L. Williamson, L. M. Gedvilas, and R. C. Reedy, Mater. Res. Soc. Symp. Proc. 762, A 10.2 (2003).

${ }^{9}$ Y. Xu, B. P. Nelson, L. M. Gedvilas, and R. C. Reedy, Thin Solid Films 430, 197 (2003).

${ }^{10}$ H. B. Stuhrmann, Adv. Polym. Sci. 67, 123 (1985).

${ }^{11}$ G. Goerigk, R. Schweins, K. Huber, and M. Ballauff, Europhys. Lett. 66, 331 (2004).

${ }^{12}$ O. Glatter and O. Kratky, Small-Angle X-ray Scattering (Academic, London 1982).

${ }^{13}$ H.-G. Haubold et al., Rev. Sci. Instrum. 60, 1943 (1989).

${ }^{14}$ D. T. Cromer and D. Liberman, J. Chem. Phys. 53, 1891 (1970).

${ }^{15}$ D. T. Cromer and D. Liberman, Acta Crystallogr., Sect. A: Cryst. Phys., Diffr., Theor. Gen. Crystallogr. 37, 267 (1981).

${ }^{16} \mathrm{G}$. Beaucage, J. Appl. Crystallogr. 28, 717 (1995).

${ }^{17}$ In the case of voids the electron density contrast simply is the electron density of the surrounding material with negative sign, while in the case of a $\mathrm{H}$ cluster it is the difference between the atomic number density of $\mathrm{H}$ atoms minus the electron density of the surrounding material. Due to only one electron of $\mathrm{H}$ the values are nearly the same. 